\title{
Hepatoprotective and Antioxidant Properties of a Triherbal Formulation against Carbon Tetrachloride Induced Hepatotoxicity
}

\author{
*O. Iroanya ${ }^{\mathrm{a}}, \mathrm{J}$. Okpuzor $^{\mathrm{b}}, \mathrm{O}$. Adebesin ${ }^{\mathrm{c}}$ \\ ${ }^{a}$ Department of Cell Biology and Genetics, University of Lagos, Akoka - Yaba, Lagos, Nigeria. \\ ${ }^{b}$ Department of Cell Biology and Genetics, University of Lagos, Akoka - Yaba, Lagos, Nigeria. \\ ${ }^{c}$ Department of Cell Biology and Genetics, University of Lagos, Akoka - Yaba, Lagos, Nigeria.
}

\begin{abstract}
The hepatoprotective activity of a triherbal formulation (GOV) comprising of $50 \%$ ethanolic extract of Gongronema latifolia, Ocimum gratissimum and Vernonia amygdalina was studied using Wistar albino rats. The animals received a single intraperitoneal injection of $\mathrm{CCl}_{4}$ in a dose of $1 \mathrm{ml} / \mathrm{kg}$ of a $50 \%(\mathrm{v} / \mathrm{v})$ solution in liquid paraffin. GOV dose dependently and significantly $(\mathrm{p}<0.05)$ attenuated the increase in serum hepatic enzyme levels after $\mathrm{CCl}_{4}$ treatment compared to the toxin control group. On administration of GOV, it dose dependently resulted in a significant $(p<0.05)$ elevation in the levels of serum CAT, GPx, GSH, GST, SOD and total protein and significant $(\mathrm{p}<0.05)$ decrease in lipid peroxidation compared to the toxin control group. It also increased the attenuated the total protein concentrations compared to the toxin control group. The results were comparable to Liv 52 and silymarin. The results of this study confirmed the antioxidant and hepatoprotective activity of the triherbal formulation against carbon tetrachloride induced nephro-hepatotoxicity in Wistar albino rats.
\end{abstract}

Key words: Carbon tetrachloride, Gongronema latifolia, Hepatoprotective, Ocimum gratissimum and Vernonia amygdalina.

\section{Introduction}

Medicinal plants are rich source of novel drugs that forms the elements in traditional systems of medicine, modern medicines, nutraceuticals, food supplements, folk medicines, pharmaceutical intermediates, bioactive principles and main compounds in synthetic drugs. Plants have long served as a useful and natural source of therapeutic agents. Almost all plants have medicinal values and their uses differ from place to place.

Liver diseases pose an enormous health problem in spite of tremendous strides in modern medicine. There is hardly any drug that can effectively control inflammation, protect the liver from the damaging effects of hydrophobic bile acids which are retained in cholestatic disorders, promote protein synthesis, manifest antioxidative, anti-lipid peroxidative and antifibrotic properties, prevent fat from infiltrating the liver, enhance glucuronidation, decreases intestinal absorption and suppresses hepatic synthesis and storage of cholesterol, stabilize hepatocyte membranes, help the liver to replace damaged tissue and regenerate itself, promotes effective metabolism of drugs by maintaining levels of CYP or Cytochrome P450, protect the liver from damage and also regulate the liver enzymes. Because of the affordability, availability and accessibility of dependable hepatoprotective drugs in scientific/ conventional medicine, plants play an important role in the management of various liver disorders and in meeting the demands of primary health care in many developing countries [1].

Gongronema latifolia, Ocimum gratissimum and Vernonia amygdalina are medicinal plants used for centuries in the African traditional medicine. These plants are commonly used in foods as vegetables and spices for flavouring and in medicine they are used for the treatment of various ailments such as diarrhea, headache, fever, hepatitis, dysentery, malaria, nausea, diabetes among others in the South Eastern part of Nigeria. They have been used singly and in combination with other plants for treatment of different disorders. The hepatoprotective potentials of a triherbal formulation containing these three plants are evaluated using carbon tetrachloride.

\section{Research Materials and Methods}

\subsection{Plant Materials}

Fresh leaves of Gongronema latifolia, Ocimum gratissimum and Vernonia amygdalina were purchased from Oyingbo market in Lagos metropolis during the rainy season (May 2007). They were subsequently identified and authenticated at the Department of Pharmacognosy, College of Medicine of the University of Lagos, Nigeria where voucher specimen (PCGH 444, PCGH 443 and PCGH 432 respectively) were deposited. The leaves were air dried at room temperature and finely ground using Corona ${ }^{\circledR}$ hand grinder. 


\subsection{Extraction of Plant Materials}

$3 \mathrm{~kg}$ of each of the powdered leaves of G. latifolia, O. gratissimum and V. amygdalina (1:1:1) was mixed and soaked in thirty litres $(30 \mathrm{~L})$ of $50 \%$ ethanol (v/v) for $24 \mathrm{hrs}$. It was filtered using three layers of cheese cloth. The solvent was removed by rotary evaporation under reduced pressure at temperatures below $45^{\circ} \mathrm{C}$ while the water was removed by freeze-drying.

\subsection{Drug Treatment Protocol for Carbon Tetrachloride Induced Hepatotoxicity}

Animals were randomized and divided into seven groups of seven animals each. Group 1 served as normal control received distilled water $\left(10 \mathrm{ml} \mathrm{kg}\right.$ body weight p.o.), group 2 served as toxin control received distilled water $\left(10 \mathrm{ml} \mathrm{kg}^{-1}\right.$ body weight p.o.), group 3-5 were treated with 2, 4 and $8 \mathrm{~g} \mathrm{~kg}^{-1}$ body weight of triherbal extract (GOV), group 6 received LIV 52 syrup (300 mg kg-1 body weight) while group 7 received Silymarin (300 $\mathrm{mg} \mathrm{kg}^{-1}$ body weight in distilled water p.o.) for fourteen days. Groups 2-7 received single intraperitoneal injection of $\mathrm{CCl}_{4}$ in a dose of $1 \mathrm{ml} \mathrm{kg}^{-1}$ of a $50 \%(\mathrm{v} / \mathrm{v})$ solution in liquid paraffin after $6 \mathrm{hr}$ of last dose administration.

\subsection{Biochemical and Antioxidant Assays}

After the experimental period, animals were anaesthetized mildly with ether and blood was collected from the retroorbital plexus. They were sacrificed and more blood samples were collected by cardiac puncture. The blood samples were used for biochemical and antioxidant studies. The livers were also dissected out for assay of oxidative stress and histology.

About one gram of the perfused liver of each animal was washed with isotonic solution. The liver homogenate was prepared using phosphate buffer solution and centrifuged. The supernatant was collected and used to assay for the effects of oxidative stress.

Serum Total protein (TP) [2], Lactate dehydrogenase (LDH) [3, 4], Gamma-glutamyl transferase (GGT) [5], Aspartate amino transferase (AST), Alanine amino transferase (ALT) and Alkaline Phosphatase (ALP) [6], activities were determined on serum. Thiobarbituric acid reactive substances (TBARS) [7], reduced glutathione (GSH) [8], Superoxide dismutase (SOD) [9], glutathione peroxidase (GPX) [10], glutathione-s-transferase (GST) [11] and Catalase (CAT) [12] activities were determined using these marker enzymes on the liver homogenate and serum samples

\subsection{Statistical analysis}

The results were expressed as Mean \pm SEM for seven rats. Statistical analysis of the data was performed using ANOVA statistical SPSS package (15.0) version. The significance of differences among all groups was determined by the Tukey HSD test. $\mathrm{P}$ - values less than $0.05(\mathrm{p} \leq 0.05)$ were considered to be statistically significant.

\section{Results}

\subsection{Effect of GOV on Serum Hepatic Enzymes on CCl4 intoxicated rats}

The effect of GOV on serum hepatic enzymes on $\mathrm{CCl}_{4}$ intoxicated rats are shown in Table 1. Pretreatment with GOV at a dose of $8 \mathrm{~g} \mathrm{~kg}^{-1}$ decreased the activity of ALP compared to Liv 52 group. At a dose of $4 \mathrm{~g} \mathrm{~kg}^{-1}$, GOV significantly $(\mathrm{p} \leq 0.05)$ decreased serum ALT activity compared to the $8 \mathrm{~g} \mathrm{~kg}^{-1}$ group and also its activity compared to Liv 52 and silymarin groups. There was a significant increase in AST activity of GOV at a dose of $8 \mathrm{~g} \mathrm{~kg}^{-1}$ compared to silymarin and control groups. GOV at a dose of $2 \mathrm{~g} \mathrm{~kg}^{-1}$ decreased the level of total bilirubin compared to all the other experimental groups and it was significant $(\mathrm{p} \leq 0.05)$ compared to the $8 \mathrm{~g} \mathrm{~kg}^{-1}$ and toxin control groups. At 2 and $8 \mathrm{~g} \mathrm{~kg}^{-1}$, GOV exhibited a decrease in GGT activities compared to Liv 52 group. The LDH activity of the Liv 52 group was increased compared to $\mathrm{GOV}$ at 4 and $8 \mathrm{~g} \mathrm{~kg}^{-1}$. 
Table 1: Serum levels of ALP, ALT, AST, GGT, LDH and TP in rats pretreated GOV before $\mathrm{CCl}_{4}$ damage.

\begin{tabular}{|c|c|c|c|c|c|c|c|c|c|c|c|c|c|}
\hline \multirow{2}{*}{\multicolumn{2}{|c|}{ Groups }} & \multirow{2}{*}{$\begin{array}{l}\text { Dose } \\
\left(\mathrm{g} \mathrm{kg}^{-1}\right)\end{array}$} & \multicolumn{11}{|c|}{ LIVER FUNCTION ENZYMES } \\
\hline & & & $\begin{array}{l}\text { ALP } \\
(\mathbf{U} / \mathbf{L})\end{array}$ & $\begin{array}{l}\text { ALT } \\
(\mathbf{U} / \mathbf{L})\end{array}$ & & $\begin{array}{l}\text { AST } \\
(\mathbf{U} / \mathbf{L})\end{array}$ & & $\begin{array}{l}\text { GGT } \\
\text { (U/L) }\end{array}$ & & $\begin{array}{l}\text { LDH } \\
\text { (U/L) }\end{array}$ & & $\begin{array}{l}\text { TP } \\
(\mathrm{g} / \mathrm{L})\end{array}$ & \\
\hline $\begin{array}{l}\text { Control } \\
\text { (Grp 1) }\end{array}$ & & & $30.5 \pm 0.88^{(\mathrm{b}, \mathrm{c})}$ & $16.4 \pm 0.8^{(b,}$ & & $\underset{\mathrm{d}, \mathrm{e})}{11.83 \pm 1^{(\mathrm{t}}}$ & & $\begin{array}{l}122.38 \\
6.37^{(\mathrm{b})}\end{array}$ & \pm & $\begin{array}{l}8.29 \\
0.49^{(b)}\end{array}$ & \pm & $\begin{array}{l}98.85 \\
5.03^{(b)}\end{array}$ & \pm \\
\hline $\begin{array}{l}\text { Toxin } \\
\text { (Grp 2) }\end{array}$ & Control & & $\underset{\mathrm{c}, \mathrm{d}, \mathrm{e})}{92.93} \pm 2.94^{(\mathrm{a},}$ & $\begin{array}{l}74.23 \\
3.22^{(\mathrm{a}, \mathrm{c}, \mathrm{d}, \mathrm{e})}\end{array}$ & & $\begin{array}{l}76.69 \\
5.29^{(\mathrm{a}, \mathrm{c}, \mathrm{d}, \mathrm{e}}\end{array}$ & \pm & $\begin{array}{l}236.5 \\
10.91^{(\mathrm{a}, \mathrm{c}, \mathrm{d},}\end{array}$ & \pm & $\begin{array}{l}34.18 \\
2.19^{(a, c, d, e}\end{array}$ & e) \pm & $\begin{array}{l}53.84 \\
3.07^{(\mathrm{a}, \mathrm{c}, \mathrm{d}, \mathrm{e}}\end{array}$ & \pm \\
\hline $\begin{array}{l}\text { GOV } \\
\text { (Grp 3) }\end{array}$ & $+\mathrm{CCl}_{4}$ & 2 & $49.81 \pm 6.49^{(b)}$ & $\begin{array}{l}34.16 \\
2.78^{(\mathrm{a}, \mathrm{b})}\end{array}$ & \pm & $\begin{array}{l}28.46 \\
0.85^{(a, b)}\end{array}$ & \pm & $\begin{array}{l}149.59 \\
10.45^{(b)}\end{array}$ & \pm & $12.47 \pm 1^{(t)}$ & & $\begin{array}{l}83.38 \\
3.62^{(\mathrm{b})}\end{array}$ & \pm \\
\hline$(\operatorname{Grp} 4)$ & & 4 & $43.32 \pm 1.45^{(\mathrm{b})}$ & $26.76 \pm .25$ & & $\begin{array}{l}27.53 \\
1.17^{(a, b)}\end{array}$ & \pm & $\begin{array}{l}161.54 \\
13.05^{(b)}\end{array}$ & \pm & $\begin{array}{l}11.64 \\
0.88^{(b)}\end{array}$ & \pm & $\begin{array}{l}85.21 \\
2.88^{(\mathrm{b})}\end{array}$ & \pm \\
\hline (Grp 5) & & 8 & $40.43 \pm 4.79^{(b)}$ & $\begin{array}{l}40.48 \\
4.13^{(\mathrm{a}, \mathrm{b}, \mathrm{d})}\end{array}$ & \pm & $\begin{array}{l}37.67 \\
5.79^{(a, b)}\end{array}$ & \pm & $\begin{array}{l}146.57 \\
11.05^{(b)}\end{array}$ & \pm & $\begin{array}{l}10.59 \\
1.69^{(\mathrm{b})}\end{array}$ & \pm & $\begin{array}{l}83.25 \\
3.76^{(\text {b) }}\end{array}$ & \pm \\
\hline $\begin{array}{l}\text { LIV } 52 \\
\text { (Grp 6) }\end{array}$ & $+\mathrm{CCl}_{4}$ & 0.3 & $42.48 \pm 1.55^{(\mathrm{b})}$ & $\begin{array}{l}34.98 \\
3.39^{(\mathrm{a}, \mathrm{b})}\end{array}$ & \pm & $\begin{array}{l}25.85 \\
2.4^{(\mathrm{b})}\end{array}$ & \pm & $\begin{array}{l}153.99 \\
10.52^{(b)}\end{array}$ & \pm & $\begin{array}{l}11.69 \\
1.27^{(\mathrm{b})}\end{array}$ & \pm & $\begin{array}{l}84.97 \\
3.64^{(\mathrm{b})}\end{array}$ & \pm \\
\hline $\begin{array}{l}\text { Silymari } \\
\text { CCl } \\
\text { (Grp 7) }\end{array}$ & in & 0.3 & $36.09 \pm 2.5^{(\mathrm{b})}$ & $\begin{array}{l}30.27 \\
2.43^{(\mathrm{a}, \mathrm{b})}\end{array}$ & \pm & $\begin{array}{l}22.23 \\
1.45^{(\mathrm{b}, \mathrm{e})}\end{array}$ & \pm & $\begin{array}{l}132.79 \\
14.68^{(b)}\end{array}$ & \pm & $9.35 \pm .93$ & & $\begin{array}{l}85.76 \\
3.32^{(\mathrm{b})}\end{array}$ & \pm \\
\hline
\end{tabular}

Values are expressed as Mean \pm SEM for seven rats. The Mean difference is significant at the .05 level. (a) $=p \leq 0.05$ as compared with the normal control group. (b) $=\mathrm{p} \leq 0.05$ as compared to $\mathrm{CCl}_{4}$ control group. (c) $=\mathrm{p} \leq 0.05$ as compared with the $\mathrm{GOV}+\mathrm{CCl}_{4}\left(2 \mathrm{~g} \mathrm{~kg}^{-1}\right)$ group. (d) $=\mathrm{p} \leq 0.05$ as compared with the $\mathrm{GOV}+\mathrm{CCl}_{4}\left(4 \mathrm{~g} \mathrm{~kg}^{-1}\right)$ group. (e) $=\mathrm{p} \leq 0.05$ as compared with the $\mathrm{GOV}+\mathrm{CCl}_{4}\left(8 \mathrm{~g} \mathrm{~kg}^{-1}\right)$ group. The significance of differences among all groups was determined by the Tukey HSD test.

ALP $=$ Alkaline phosphatase, ALT $=$ Alanine aminotransferase, AST $=$ aspartate aminotransferase, GGT $=$ L- $\gamma-$ glutamyltransferase and LDH = Lactate dehydrogenase

\subsection{The effect of $\mathbf{C C l}_{4}$ damage on serum antioxidant enzymes in rats pretreated with GOV}

The activities of antioxidant enzymes CAT, GPx, GSH, GST, MDA, SOD and total protein after intraperitoneal administration of $\mathrm{CCl}_{4}$ are depicted in Tables 2. It is observed that, GOV administration resulted in a significant $(\mathrm{p} \leq 0.05)$ elevation in the levels of CAT, GPx, GSH, GST, SOD and total protein and significant $(\mathrm{p} \leq 0.05)$ decrease in lipid peroxidation compared to the toxin control group. Compared to Liv 52 and silymarin, administration of GOV at $4 \mathrm{~g} \mathrm{~kg}^{-1}$ exhibited almost the same CAT and SOD activities while the GSH activity was higher. GOV at a dose of $8 \mathrm{~g} \mathrm{~kg}^{-1}$ and Liv 52 group has the same GSH activities. Pretreatment with GOV at $4 \mathrm{~g} \mathrm{~kg}^{-1}$ exhibited higher GSH activity compared to Liv 52 and silymarin groups while the MDA activity was lower than that exhibited by Liv 52. It also showed almost the same SOD activity compared to Liv 52 and silymarin. GOV at a dose of $2 \mathrm{~g} \mathrm{~kg}^{-1}$ showed significantly ( $\left.\mathrm{p} \leq 0.05\right)$ attenuated CAT, GSH and SOD activities compared to control, Liv 52 , silymarin and GOV at $4 \mathrm{~g} \mathrm{~kg}^{-1}$ groups. 
Table 2: The effect of $\mathrm{CCl}_{4}$ damage on serum antioxidant enzymes in rats pretreated with GOV

\begin{tabular}{|c|c|c|c|c|c|c|c|c|}
\hline Groups & $\begin{array}{l}\text { Dose } \\
(\mathrm{g} \mathrm{kg} \\
\left.{ }_{1}\right)\end{array}$ & $\begin{array}{l}\text { CAT } \\
(\mu \mathrm{mol} / \mathrm{min} / \mathrm{mg} \\
\text { protein) }\end{array}$ & $\begin{array}{l}\text { GPx } \\
(\mu \mathrm{mol} / \mathrm{ml})\end{array}$ & $\begin{array}{l}\text { GSH } \\
(\mu \mathrm{mol} / \mathrm{ml})\end{array}$ & $\begin{array}{l}\text { GST } \\
(\mu \mathrm{mol} / \mathrm{ml})\end{array}$ & $\begin{array}{l}\text { MDA } \\
(\mathbf{n m o l} / \mathrm{ml})\end{array}$ & $\begin{array}{l}\text { SOD } \\
(\mu \mathrm{mol} / \mathrm{ml})\end{array}$ & $\begin{array}{l}\text { TP } \\
(\mathrm{g} / \mathrm{l})\end{array}$ \\
\hline $\begin{array}{l}\text { Control } \\
\text { (Grp 1) }\end{array}$ & & $\underset{\mathrm{d}, \mathrm{e})}{87.41} \pm 3.36^{(\mathrm{b}, \mathrm{c}}$ & $\begin{array}{l}14.96 \\
1.11^{(\mathrm{b}, \mathrm{c}, \mathrm{d}, \mathrm{e})}\end{array}$ & 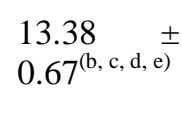 & $\begin{array}{l}126.46 \\
1.66^{(\mathrm{b}, \mathrm{c}, \mathrm{d}, \mathrm{e})}\end{array}$ & $\begin{array}{l}4.92 \\
0.1^{(\mathrm{b}, \mathrm{c}, \mathrm{d}, \mathrm{e})}\end{array}$ & $\begin{array}{l}92.13 \\
3.4^{(\mathrm{b}, \mathrm{c}, \mathrm{d}, \mathrm{e})}\end{array}$ & $\begin{array}{l}98.85 \pm \\
5.03^{(\mathrm{b}, \mathrm{c}, \mathrm{d}}, \\
\mathrm{e})\end{array}$ \\
\hline $\begin{array}{l}\text { Toxin Control } \\
\text { (Grp 2) }\end{array}$ & & $\underset{\mathrm{d}, \mathrm{e})}{22.22} \pm 0.93^{(\mathrm{a}, \mathrm{c}}$ & $\begin{array}{l}2.46 \\
0.01^{(\mathrm{a}, \mathrm{c}, \mathrm{d}, \mathrm{e})}\end{array}$ & $\underset{(\mathrm{a}, \mathrm{c}, \mathrm{d}, \mathrm{e})}{4.27 \pm 0.2}$ & $\begin{array}{l}39.23 \pm \\
1.76^{(\mathrm{a}, \mathrm{c}, \mathrm{d}, \mathrm{e})}\end{array}$ & $\begin{array}{l}16.29 \underset{(\mathrm{a}, \mathrm{c}, \mathrm{d}, \mathrm{e})}{ \pm} \\
0.6{ }^{2}\end{array}$ & $\begin{array}{l}21.21 \pm \\
1.27^{(\mathrm{a}, \mathrm{c}, \mathrm{d}, \mathrm{e})}\end{array}$ & $\begin{array}{l}53.84 \quad \pm \\
3.07 \\
\mathrm{~d}, \mathrm{e})\end{array}$ (a, $\mathrm{c}$ \\
\hline$\underset{(\mathrm{Grp} \mathrm{3})}{\mathrm{GOV}}+\mathrm{CCl}_{4}$ & 2 & 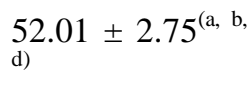 & $\begin{array}{l}8.65 \\
0.39^{(\mathrm{a}, \mathrm{b})}\end{array} \quad \pm$ & $\begin{array}{l}7.14 \\
0.4^{(\mathrm{a}, \mathrm{b}, \mathrm{d}, \mathrm{e})}\end{array}$ & $\begin{array}{l}89.86 \\
8.54^{(\mathrm{a}, \mathrm{b})}\end{array} \quad \pm$ & $\begin{array}{ll}9.2 & \pm \\
0.47^{(\mathrm{a}, \mathrm{b})} & \end{array}$ & $\begin{array}{l}55.89 \\
2.81_{(\mathrm{a}, \mathrm{b}, \mathrm{d})}^{ \pm}\end{array}$ & $\begin{array}{l}74.8 \\
1.3^{(\mathrm{a}, \mathrm{b})}\end{array}$ \\
\hline (Grp 4) & 4 & $\underset{c)}{67.13} \pm 1.95^{(\mathrm{a}, \mathrm{b}}$ & $\begin{array}{l}9.26 \\
0.24^{(\mathrm{a}, \mathrm{b})}\end{array} \quad \pm$ & $\begin{array}{l}10.25 \\
0.24^{(\mathrm{a}, \mathrm{b}, \mathrm{c})}\end{array} \pm$ & $\begin{array}{l}97.38 \\
3.04^{(a, b)}\end{array} \pm$ & $\begin{array}{l}7.55 \\
0.32^{(\mathrm{a}, \mathrm{b})}\end{array}$ & $\begin{array}{l}77.6 \\
1.85^{(\mathrm{a}, \mathrm{b}, \mathrm{c})}\end{array} \pm$ & $\begin{array}{l}79.49 \pm \\
1.77^{(\mathrm{a}, \mathrm{b})}\end{array}$ \\
\hline (Grp 5) & 8 & $61.14 \pm 1.88^{(\mathrm{a}, \mathrm{b})}$ & $\begin{array}{l}9.38 \\
0.55^{(\mathrm{a}, \mathrm{b})}\end{array} \quad \pm$ & $\begin{array}{l}9.8 \\
0.34^{(\mathrm{a}, \mathrm{b}, \mathrm{c})}\end{array}$ & $\begin{array}{l}92.12 \\
4.96^{(\mathrm{a}, \mathrm{b})}\end{array} \pm$ & $\underset{(a, b)}{8.68} \pm 0.15$ & $\begin{array}{l}66.61 \\
3.31^{(\mathrm{a}, \mathrm{b})}\end{array}$ & $\begin{array}{l}77.71 \pm \\
1.21^{(\mathrm{a}, \mathrm{b})}\end{array}$ \\
\hline $\begin{array}{l}\operatorname{LIV~52}+\mathrm{CCl}_{4} \\
(\text { Grp 6) }\end{array}$ & 0.3 & ${ }_{\mathrm{c})}^{68.23} \pm 3.53^{(\mathrm{a}, \mathrm{b}}$ & $\begin{array}{l}9.49 \\
0.5^{(\mathrm{a}, \mathrm{b})}\end{array}$ & $\begin{array}{l}9.8 \\
0.34^{(\mathrm{a}, \mathrm{b}, \mathrm{c})}\end{array}$ & $\begin{array}{l}102.84 \\
3.64^{(a, b)}\end{array}$ & $\underset{(a, b)}{7.71} \pm 0.46$ & $\begin{array}{l}78.62 \pm \pm \\
1.42^{(\mathrm{a}, \mathrm{b}, \mathrm{c}, \mathrm{e})}\end{array}$ & $\begin{array}{l}82.11 \\
2.74^{(a, b)}\end{array} \pm$ \\
\hline $\begin{array}{l}\text { Silymarin } \\
\text { CCl }_{4} \\
\text { (Grp 7) }\end{array}$ & 0.3 & 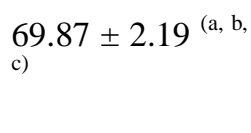 & $\begin{array}{l}10.58 \\
0.51^{(\mathrm{a}, \mathrm{b})}\end{array} \quad \pm$ & $\begin{array}{l}10.04 \\
0.33^{(\mathrm{a}, \mathrm{b}, \mathrm{c})}\end{array} \pm$ & $\begin{array}{l}111.02 \\
7.46^{(b)}\end{array}$ & $\begin{array}{l}6.86 \pm 4^{(\mathrm{a}, \mathrm{b}, \mathrm{c}, \mathrm{e})} \\
\mathbf{1}\end{array}$ & $\begin{array}{l}79.88 \pm \pm \\
2.89^{(\mathrm{a}, \mathrm{b}, \mathrm{c}, \mathrm{e})}\end{array}$ & $\begin{array}{l}81.24 \pm \\
2.08^{(\mathrm{a}, \mathrm{b})}\end{array}$ \\
\hline
\end{tabular}

Values are expressed as Mean \pm SEM for seven rats. The Mean difference is significant at the .05 level. (a) $=p \leq 0.05$ as compared with the normal control group. (b) $=\mathrm{p} \leq 0.05$ as compared to $\mathrm{CCl} 4$ control group. (c) $=\mathrm{p} \leq 0.05$ as compared with the GOV + CCl4 (2 $\left.\mathrm{g} \mathrm{kg}^{-1}\right)$ group. (d) $=\mathrm{p} \leq 0.05$ as compared with the GOV + $\mathrm{CCl} 4\left(4 \mathrm{~g} \mathrm{~kg}^{-1}\right)$ group. (e) $=\mathrm{p} \leq 0.05$ as compared with the GOV + $\mathrm{CCl} 4\left(8 \mathrm{~g} \mathrm{~kg}^{-1}\right)$ group. The significance of differences among all groups was determined by the Tukey HSD test.

Results presented in Table 3 indicates that the elevated level of MDA due to $\mathrm{CCl}_{4}$ intoxication were significantly $(p \leq 0.05)$ attenuated in rats, after treatment with GOV. GOV exhibited lower MDA activity compared the standard reference drug groups. The GOV pretreated rats in the $4 \mathrm{~g} \mathrm{~kg}^{-1}$ group, showed increased GPx activity compared to the standard reference drugs. It also showed significantly $(\mathrm{p} \leq 0.05)$ decreased SOD activity compared to the control group. At $2 \mathrm{~g} \mathrm{~kg}^{-1}$ and $8 \mathrm{~g} \mathrm{~kg}^{-1}$, GOV showed significant $(\mathrm{p} \leq 0.05)$ decrease in the concentration of total protein compared to the standard reference drugs. The catalase activity of GOV was significantly $(\mathrm{p} \leq 0.05)$ decreased compared to control and standard reference drug groups. 
Table 3: The effect of $\mathrm{CCl}_{4}$ damage on liver antioxidant enzymes in rats pretreated with GOV

\begin{tabular}{|c|c|c|c|c|c|c|c|c|c|c|c|c|}
\hline Groups & $\begin{array}{l}\text { Dose } \\
\text { (g kg } \\
\text { 1) }\end{array}$ & $\begin{array}{l}\text { CAT } \\
(\mu \mathrm{mol} / \mathrm{min} / \mathrm{mg} \\
\text { protein) }\end{array}$ & $\begin{array}{l}\text { GPx } \\
(\mu \mathrm{mol} / \mathrm{m}\end{array}$ & & $\begin{array}{l}\text { GSH } \\
(\mu \mathrm{mol} / \mathrm{ml})\end{array}$ & & $\begin{array}{l}\text { GST } \\
(\mu \mathrm{mol} / \mathrm{ml})\end{array}$ & & $\begin{array}{l}\text { MDA } \\
(\mathbf{n m o l} / \mathbf{m l}\end{array}$ & & $\begin{array}{l}\text { SOD } \\
(\mu \mathrm{mol} / \mathrm{ml})\end{array}$ & $\begin{array}{l}\mathbf{T P} \\
(\mathrm{g} / \mathrm{l})\end{array}$ \\
\hline $\begin{array}{l}\text { Control } \\
\text { (grp 1) }\end{array}$ & & $\begin{array}{l}210.73 \\
10.96^{(\mathrm{b}, \mathrm{c}, \mathrm{d}, \mathrm{e})}\end{array} \pm$ & $\begin{array}{l}55.12 \\
4.49^{(\mathrm{b})}\end{array}$ & \pm & $\begin{array}{l}137.53 \\
14.2^{(\mathrm{b}, \mathrm{d}, \mathrm{e})}\end{array}$ & \pm & $\begin{array}{l}296.91 \\
31.32^{(b)}\end{array}$ & \pm & $\begin{array}{l}13.64 \\
1.27^{(\mathrm{b})}\end{array}$ & \pm & $\begin{array}{l}106.65 \\
4.04^{(\mathrm{b}, \mathrm{c}, \mathrm{d}, \mathrm{e})}\end{array}$ & $\begin{array}{l}114.79 \pm \\
5.06^{(b, c, d},\end{array}$ \\
\hline $\begin{array}{l}\text { Toxin Control } \\
\text { (Grp 2) }\end{array}$ & & $\underset{\mathrm{d}, \mathrm{e})}{69.8 \pm 5.89^{(\mathrm{a}, \mathrm{c},}}$ & $\begin{array}{l}28.57 \\
.99^{(\mathrm{a}, \mathrm{c}, \mathrm{d}, \mathrm{e}}\end{array}$ & \pm & $\begin{array}{l}58.94 \\
4.77^{(\mathrm{a}, \mathrm{c}, \mathrm{e})}\end{array}$ & \pm & $\begin{array}{l}67.86 \\
5.12^{(\mathrm{a}, \mathrm{c}, \mathrm{d}, \mathrm{e}}\end{array}$ & \pm & $\begin{array}{l}36.35 \\
3.37^{(a, c, d},\end{array}$ & \pm & 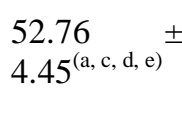 & $\begin{array}{l}56.07 \pm \\
2.73^{(\mathrm{a}, \mathrm{c}, \mathrm{d}} \\
\mathrm{e})\end{array}$ \\
\hline$\underset{(\mathrm{Grp} \mathrm{3})}{\mathrm{GOV}}+\mathrm{CCl}_{4}$ & 2 & b) $148.03 \pm 1.6^{(\mathrm{a},}$ & $\begin{array}{l}43.37 \\
1.31^{(\mathrm{b})}\end{array}$ & \pm & $\begin{array}{l}91.06 \\
2.28^{(\mathrm{a}, \mathrm{b})}\end{array}$ & \pm & $\begin{array}{l}222.45 \\
15.86^{(b)}\end{array}$ & \pm & $\begin{array}{l}15.59 \\
0.89^{(b)}\end{array}$ & \pm & $\begin{array}{l}85.68 \\
2.1^{(a, b)}\end{array}$ & $\begin{array}{l}80.15 \pm \\
2.23^{(\mathrm{a}, \mathrm{b})}\end{array}$ \\
\hline (Grp 4) & 4 & $\begin{array}{l}151.98 \\
6.86^{(\mathrm{a}, \mathrm{b})}\end{array}$ & $\begin{array}{l}46.81 \\
4.01^{(\mathrm{b})}\end{array}$ & \pm & $\begin{array}{l}103.52 \\
12.21^{(\mathrm{b})}\end{array}$ & \pm & $\begin{array}{l}250.43 \\
16.89^{(\mathrm{b})}\end{array}$ & \pm & $\begin{array}{l}15.6 \\
1.71^{(b)}\end{array}$ & \pm & $\begin{array}{l}80.7 \\
2.65^{(\mathrm{a}, \mathrm{b})}\end{array}$ & $\begin{array}{l}84.25 \pm \\
3.15^{(\mathrm{a}, \mathrm{b})}\end{array}$ \\
\hline (Grp 5) & 8 & $\begin{array}{l}151.76 \\
2.07^{(\mathrm{a}, \mathrm{b})}\end{array}$ & $\begin{array}{l}44.78 \\
2.31^{(\mathrm{b})}\end{array}$ & \pm & $\begin{array}{l}100.57 \\
4.35^{(a, b)}\end{array}$ & \pm & $\begin{array}{l}262.41 \\
8.09^{(b)}\end{array}$ & \pm & $\begin{array}{l}15.14 \\
1.2^{(\mathrm{b})}\end{array}$ & \pm & $\begin{array}{l}84.63 \\
4.78^{(\mathrm{a}, \mathrm{b})}\end{array}$ & $\begin{array}{l}77.93 \pm \\
1.03^{(\mathrm{a}, \mathrm{b})}\end{array}$ \\
\hline $\begin{array}{l}\operatorname{LIV~} 52 \\
(\text { Grp 6) }\end{array}$ & 0.3 & $\begin{array}{ll}187.98 & \pm \\
7.57^{(\mathrm{b}, \mathrm{c}, \mathrm{d}, \mathrm{e})} & \pm\end{array}$ & $\begin{array}{l}45.33 \\
2.65^{(\mathrm{b})}\end{array}$ & \pm & $\begin{array}{l}115.6 \\
5.01^{(\mathrm{b})}\end{array}$ & \pm & $\begin{array}{l}296.33 \\
10.67^{(b)}\end{array}$ & \pm & $\begin{array}{l}16.39 \\
0.8^{(\mathrm{b})}\end{array}$ & \pm & $\begin{array}{l}93.89 \\
3.73^{(\text {b) }}\end{array}$ & $\begin{array}{l}98.31 \pm \\
3.94^{(\mathrm{a}, \mathrm{b}, \mathrm{c}}, \\
\mathrm{e})\end{array}$ \\
\hline $\begin{array}{l}\text { Silymarin } \\
\text { CCl } \\
\text { (Grp 7) }\end{array}$ & 0.3 & $\begin{array}{l}190.45 \\
5.81^{(\mathrm{b}, \mathrm{c}, \mathrm{d}, \mathrm{e})}\end{array} \quad \pm$ & $\begin{array}{l}45.71 \\
2.21^{(\mathrm{b})}\end{array}$ & \pm & $\begin{array}{l}117.54 \\
4.52^{(b)}\end{array}$ & \pm & $\begin{array}{l}287.64 \\
35.15^{(b)}\end{array}$ & \pm & $\underset{\text { (b) }}{16.56 \pm 0}$ & & $\begin{array}{l}98.28 \\
3.19^{(\mathrm{b}, \mathrm{c})}\end{array}$ & $\begin{array}{l}95.03 \quad \pm \\
1.96 \\
\mathrm{c}, \mathrm{e})\end{array}$ \\
\hline
\end{tabular}

Values are expressed as Mean \pm SEM for seven rats. The Mean difference is significant at the .05 level. (a) $=p \leq 0.05$ as compared with the normal control group. (b) $=\mathrm{p} \leq 0.05$ as compared to CCl4 control group. (c) $=\mathrm{p} \leq 0.05$ as compared with the GOV + CCl4 (2 g kg-1) group. (d) $=\mathrm{p} \leq 0.05$ as compared with the GOV + CCl4 (4 g kg-1) group. (e) $=\mathrm{p} \leq 0.05$ as compared with the GOV + CCl4 (8g kg-1) group. The significance of differences among all groups was determined by the Tukey HSD test.

CAT $=$ Catalase, GPx $=$ Glutathione Peroxidase, GSH $=$ Reduced Glutathione, GST $=$ Glutathione-STransferase, $\mathrm{MDA}=$ Malondialdehyde, $\mathrm{SOD}=$ Superoxide Dismutase, $\mathrm{TP}=$ Total Protein

\section{Discussions}

The experimentally induced cirrhotic response by carbon tetrachloride $\left(\mathrm{CCl}_{4}\right)$ in rats and mice has been shown to be similar to human cirrhosis of the liver [13]. $\mathrm{CCl}_{4} \mathrm{can}$ induce liver damage through the formation of reactive free radicals that can bind covalently to cellular macromolecules forming nucleic acid, protein and lipid adducts, through the induction of hypomethylated ribosomal RNA, resulting in inhibition of protein synthesis and it can also affect hepatocellular calcium homeostasis $[14,15] . \mathrm{CCl}_{4}$ treatment can result in centrilobular steatosis, inflammation, apoptosis and necrosis $[14,15,16]$. The liver will progress to fibrosis and cirrhosis if the damage exceeds the ability of the liver to repair [15].

Mansour reported that a single dose of $\mathrm{CCl}_{4}$ induced hepatotoxicity manifested biochemically by significant elevation of activities of liver functions, such as ALT and AST [17]. The results of the present study shows that GOV at different doses $\left(2,4\right.$ and $\left.8 \mathrm{~g} \mathrm{~kg}^{-1}\right)$ significantly $(\mathrm{P}<0.05)$ protected the rats against $\mathrm{CCl}_{4}$-induced hepatotoxicity as demonstrated by its inhibition of the elevation of serum AST ALT, ALP, GGT, total bilirubin and LDH activities. $\mathrm{CCl}_{4}{ }^{-}$ induced acute liver damage may be initiated by the trichloromethyl free radical, which is formed by cytochrome $\mathrm{P} 450$ and 
could induce peroxidation of the unsaturated fatty acids of cell membrane. This leads to membrane injury and leakage of enzymes e.g. AST and ALT [18]. Aminotransferase levels are sensitive indicators of liver-cell injury and are helpful in recognizing hepatocellular diseases while gamma glutamyl transferase a sensitive indicator of cholestasis is useful to help determine if elevations of alkaline phosphatase are due to bone or liver disorder.

The main mechanism by which $\mathrm{CCl}_{4}$ is known to mediate its toxic effects is through oxidative stress and oxidative damage due to an increased production of Reactive Oxygen Species (ROS) [19]. A variety of enzymatic and non-enzymatic mechanisms have evolved to protect cells against ROS, including the superoxide dismutases, which remove $\mathrm{O}_{2}$; catalase and the glutathione $(\mathrm{GSH})$ peroxidase system which remove $\mathrm{H}_{2} \mathrm{O}_{2}$; glutathione transferases which can remove reactive intermediates and lipid aldehydes [20,21]. GOV significantly $(\mathrm{P}<0.05)$ increased the activities of catalase, GSH, GPx, GST and superoxide dismutase but decreased the lipid peroxidation level compared to the toxin control rats. Malondialdehyde (MDA) is a cytotoxic reactive aldehyde [15] formed as a byproduct of lipid peroxidation. The ability of GOV to attenuate the elevated levels of MDA indicates it hepatoprotective potentials. Depletion of GSH has been shown to enhance CYP2E1 resulting in CYP2E1-derived ROS leading to toxicity [22]. Since CYP2E1 is a key contributor to injury produced by $\mathrm{CCl}_{4}$, one possible mechanism involved in the prevention of this toxicity by GOV could have been an inhibition of CYP2E1 catalytic activity. The mechanism of protection can be attributed to decreased production of ROS and lipid peroxidation when the CYP2E1 mediated oxidative stress was produced in hepatocytes with pro-oxidant as $\mathrm{CCl}_{4}$

Rat hepatocytes undergo apoptosis after injection of $\mathrm{CCl}_{4}$ [16]. It increased accumulation of apoptotic cells and is thought to play an important role in the progression of liver injury. A great deal of evidence indicates that cell exposure to ROS, in addition to producing direct chemical injury to cell components, also induces apoptosis, which leads to cell disruption and tissue necrosis [23].

\section{Conclusion}

This triherbal formulation (GOV) has demonstrated liver protective effect against carbon tetrachloride-induced hepatotoxicity by amelioration of the effects of $\mathrm{CCl}_{4}$ on liver function tests including serum levels of liver enzymes and antioxidant enzymes. It exhibited antioxidant activities in a dose dependent manner and demonstrated significant protection to the liver thus justifying its use as a hepatoprotective agent. These reports show that GOV can protect against $\mathrm{CCl}_{4}$-induced toxicity and oxidative stress. The present findings provide scientific evidence to the ethnomedicinal use of GOV in Eastern Nigeria in treating liver diseases.

\section{References}

1. World Health Organization (WHO). Traditional medicine strategy 2002-2005, World Health Organization, WHO/EDM/TRM/2002.1, Geneva, 2001,pp.7

2. N.W. Tietz (Ed): Clinical Guide to Laboratory Tests, 3rd ed. W. B. Saunders, Philadelphia, PA, 1995.

3. Deutsche Gesellschaft für klinische Chemie. Empfehlungen der deutschen Gesellschaft für Klinische Chemie (DGKC). Standardisierung von Methoden zur Bestimmung von Enzymaktivitäten in biologischen Flüssigkeiten. (Recommendation of the German Society of Clinical Chemistry. Standardization of methods for measurement of enzymatic activities in biological fluids.). Journal of Clinical Chemistry and Clinical Biochemistry. 8, 1970, 658.

4. Deutsche Gesellschaft für klinische Chemie. Empfehlungen der deutschen Gesellschaft für Klinische Chemie (DGKC). Standardisierung von Methoden zur Bestimmung von Enzymaktivitäten in biologischen Flüssigkeiten. (Recommendation of the German Society of Clinical Chemistry. Standardization of methods for measurement of enzymatic activities in biological fluids.) Journal of Clinical Chemistry and Clinical Biochemistry. 10, 1972, 182 92.

5. G. Szaszi. A kinetic photometric method for serum gammaglutamyl transpeptidase. Clinical Chemistry. 15 (2), 1969,124-136.

6. S. Reitman and S. Frankel. A colourimetric method for the determination of serum glutamic oxalacetic and glutamic pyruvic transaminases. American Journal of Clinical Pathology. 28 (1), 1957: 56-63.

7. H. Esterbauer and K.H., Cheeseman. Determination of aldehydic lipid peroxidation products: Malonaldhyde and 4hydroxynonetal. Methods in Enzymology. 186, 1990, 407-421.

8. J. Sedlak and R.H. Lindsay. Estimation of total, protein-bound and nonprotein sulfhydryl groups in tissue by Ellmans reagent. Analytical Biochemistry. 25 (1), 1968, 192-205.

9. P. Kakkar, B. Das and P. N. Viswanathan. A modified spectrophotometric assay of SOD. Indian Journal of Biochemistry and Biophysics. 21 (2), 1984, 130- 132.

10. D.G. Hafemann, R.A. Sunde and W.G. Houestra. Effect of dietary selenium on erythrocyte and liver glutathione peroxidase in the rat. Journal of Nutrition. 104 (5), 1974, 580-584. 
11. W. H. Habig and W. B. Jakoby. Glutathione S-Transferases. The first enzymatic step in mercapturic acid formation. The Journal of Biological Chemistry. 249 (22), 1974, 7130-7139.

12. J. Bonaventura, W. A. Schroeder and S. Fang. Human erythrocyte catalase: an improved method of isolation and a revaluation of reported properties. Archives of Biochemistry and Biophysics. 150 (2), 1972, 606-617.

13. C. Weiler-Normann, J. Herkel and A.W. Lohse. Mouse models of liver fibrosis. Zeitschrift fur Gastroenterologie. 45(1), 2007, 43-50.

14. L. W. Weber, M. Boll and A. Stampfl. Hepatotoxicity and mechanism of action of haloalkanes: carbon tetrachloride as a toxicological model. Critical Reviews in Toxicology. 33 (2), 2003, 105-136.

15. M.K. Manibusan, M. Odin and D.A. Eastmond. Postulated carbon tetrachloride mode of action: a review. Journal of Environmental Science and Health, Part C. 25 (3), 2007, 185-209.

16. J. Shi, K. Aisaki, Y. Ikawa and K. Wake. Evidence of hepatocytes apoptosis in rat liver after the administration of carbon tetrachloride. The American Journal of Pathology. 153 (2), 1998, 515-525.

17. H. H. Mansour, H. F. Hafez and N. M. Fahmy. Silymarin modulates Cisplatin-induced oxidative stress and hepatotoxicity in rats. International Journal of Biochemistry and Molecular Biology. 39 (6), 2006, 656-661.

18. C.S. Lee, J.H. Han, Y.Y. Jang, J.H. Song and E.S. Han. Differential effect of catecholamines and MMP+ on membrane permeability in brain mitochondria and cell viability in PC12 cells. Neurochemistry International. 40 (4), 2002, 361-369.

19. G. E. Arteel. Oxidants and antioxidants in alcohol-induced liver disease. Gastroenterology. 124(3), 2003, 778-790.

20. B.P. Yu. Cellular defenses against damage from reactive oxygen species. Physiological Reviews. 74 (1), 1994 , $139-162$.

21. B. Halliwell. Antioxidant defence mechanisms: from the beginning to the end (of the beginning). Free Radical Research. 31 (4), 1999, 261-272.

22. J. Zhuge and A.I. Cederbaum. Depletion of S-adenosyl-1-methionine with cycloleucine potentiates cytochrome P450 2E1 toxicity in primary rat hepatocytes. Archives of Biochemistry and Biophysics. 466 (2), 2007, 177-185.

23. T. Ozben. Oxidative stress and apoptosis: impact on cancer therapy. Journal of Pharmaceutical Sciences. 96 (9), 2007, 2181-2196. 